\title{
Supplementary motor area - clinical semiology and results of direct electrical stimulation during intracranial electrodes exploration
}

\author{
Anca Adriana Arbune, Ovidiu Alexandru Bajenaru, Irina Popa, Ioana Mindruta \\ Neurology Clinic, University Emergency Hospital, "Carol Davila” University of Medicine and Pharmacy, \\ Department 6 Clinical Neurosciences, Bucharest, Romania
}

\begin{abstract}
Objectives. We aimed to study the supplementary motor area (SMA) by observing the clinical semiology of the seizures generated by an epileptogenic zone containing the SMA and by performing direct electrical stimulation (DES) of the SMA explored by means of intracranial depth electrodes using various stimulation protocols.

Material and method. We invasively explored with intracerebral depth electrodes 65 patients with focal drug resistant epilepsy as presurgical evaluation. Only 5 had the SMA included in the epileptogenic zone (EZ). We recorded multiple spontaneous and provoked seizures. We applied specific DES (single pulse electrical stimulation - SPES and $50 \mathrm{~Hz}$ protocols) to the SMA and adjacent cerebral structures. All patients were operated on and had the EZ removed, have currently good outcomes (Engel I and II) at more than 1 year postsurgery and have no neurological deficits.

Results. All stereoelectroencephalographic (SEEG) recorded seizures indicated the SMA as part of the EZ. Common manifestations were grimacing, version of the head to one side, tonic asymmetric posturing of the upper limbs and secondary generalization. The DES of the SMA induced forced adduction of the contralateral upper limb, flexion of the trunk to the contralateral side, clonia of the contralateral lower limbs. SPES stimulation revealed SMA connectivity with various frontal and parietal structures, as well as the influence of sleep on these connections.

Discussion. SMA as part of the EZ elicited seizures with varying and complex clinical features, with little lateralizing value. DES elicited mostly motor phenomena, confirming previous studies, but no speech arrest. Of special interest was the SMA - middle cingulate cortex (MCC) relationship. Sleep seems to modulate SMA connectivity. The clinico-anatomico-electrographic correlations indicated a wide epileptogenic zone for resection, including SMA and MCC. Conclusions. The SMA is usually only a part of the frontal EZ, which usually includes the MCC other frontal mesial brain structures. Semiology of the seizures does not always provide lateralization cues and invasive exploration is needed for the delineation of the resection area. Connectivity of the SMA during wakefulness and sleep exhibits a complex relationships and further studies are needed. The SMA seems to be safe to remove on the long term.
\end{abstract}

Keywords: supplementary motor area, middle cingulate cortex, direct electrical stimulation, 50 $\mathrm{Hz}$ stimulation, SPES, sleep SPES, drug-resistant epilepsy, SEEG, epilepsy surgery

\section{INTRODUCTION}

The supplementary motor area (SMA) is part of the frontal lobe, which makes up about $40 \%$ of the brain and has extensive connectivity within and with neighboring areas. The mesial networks and orbitofrontal region are difficult to sample via scalp EEG, especially because of rapid propagation of impulses (Bonini et al., 2014).

Frontal lobe seizures are usually short, with a duration under 1 minute, occur in clusters, appear more frequently during sleep, rapidly involve the entire brain hemisphere and often spread to the contralateral side, resulting in secondary generalized seizures (Bonelli \& Baumgartner, 2002).

The SMA is known to be responsible for many functions, having important roles in movement, coordination, speech, thus the difficulties of localization using clinical semiology.

Typical SMA seizures have postural and tonic signs, predominantly proximal, usually bilateral and asymmetrical. Involvement of the upper limbs 
produces the "4 sign", "fencing posture" or other tonic postures. Head and eyes version is often associated due to the close proximity to the frontal eye fields. The direction of version can be ipsilateral or contralateral to the seizure onset area, depending on its timing within the seizure, and is not trustworthy as lateralization sign. Speech arrest or ictal vocalization may also occur, as the involvement of the SMA in language processing is documented. Facial clonic movements and hypersalivation also occur. More complex movements of all four limbs can follow these main signs. Auras are uncommon in SMA seizures, but some patients describe sensory symptoms such as poorly defined, generalized or localized, constriction or tingling. Secondary generalization is infrequent (Bonelli et al., 2007; Janszky, Fogarasi, Jokeit, \& Ebner, 2001; Saint-Hilaire \& Lee, 2000; Serles et al., 1998; Tufenkjian \& Lüders, 2012).

Direct electrical stimulation of the SMA has been conducted in a limited number of patients. First reports by Penfield in the 1950s' described speech arrest when stimulating the SMA, while further studies added negative motor effects and somatosensory elementary sensations (Chassagnon, Minotti, Kremer, Hoffmann, \& Kahane, 2008; Filevich, Kühn, \& Haggard, 2012).

\section{MATERIAL AND METHODS}

Out of 65 patients with drug resistant epilepsy, who were part of the National Romanian Epilepsy Surgery Program, we identified five patients with the supplementary motor area as seizure onset zone. All patients gave their informed consent for all the procedures related to presurgical evaluation and data processing for research.

Stereoelectroencephalographic (SEEG) exploration was considered necessary in order to plan a restricted resection due to the absence of clear-cut cerebral MRI lesions. Patients underwent invasive exploration using intracerebral depth electrodes based on an individually designed implantation chart in accordance with semiology, non-invasive video-EEG recordings and MRI findings.

We used depth electrodes (Dixi medical, Besancon France) $0.8 \mathrm{~mm}$ diameter with $8-18$ contacts, 2 $\mathrm{mm}$ in length, situated at $1.5 \mathrm{~mm}$ distance each. They were placed in stereotactic conditions with both orthogonal (Balanescu et al, 2014) and oblique trajectories. To identify the exact location of the electrodes and contacts post-implantation cerebral CT scan was performed. Then, it was coregistered with MRI T1 sequence in the initial implantation plan using Microtargeting Waypoint Planner 3.0.

For all patients, SEEG recordings were conducted for 7 to 14 days in the Epilepsy Monitoring Unit at the University Emergency Hospital Bucharest, using a 64 contacts Nicolet Wireless 64 Amplifier (Natus Medical Inc.). We obtained spontaneous seizures in all patients.

We used a specially programmed stimulation unit and software, designed by our collaborator from the Physics Faculty of the University of Bucharest. We stimulated with direct electrical current the electrode contacts situated in gray matter using specific protocols for connectivity mapping with single pulses (1 Hz, SPES) and for functional mapping with trains of $50 \mathrm{~Hz}$ pulses. We also performed signal analysis with specialized processing computer algorithms, of the SPES stimulation responses - early (first $100 \mathrm{~ms}$ after the pulse) and late (between 100 and $500 \mathrm{~ms}$ after the pulse). The main goal when performing these electrical stimulations was to observe the epileptogenic network and its connectivity. The stimulations were performed during wakefulness in all patients, and also during sleep in one patient. All the protocols are described in previous articles.

All patients underwent surgical resection of the SMA along with other brain structures involved in the identified epileptogenic network. Four patients are seizure-free at more than 1 year after the resection (Engel I), and one patient still has a few seizures (Engel II) at 1 year after the resection. No patient had long-term postsurgical deficits.

\section{RESULTS}

We recorded 84 spontaneous seizures in five patients whose epileptogenic zone (EZ) included the SMA during SEEG monitoring. We also recorded another 19 provoked seizures during $50 \mathrm{~Hz}$ cortical stimulation of the SMA and adjacent structures, all similar to the habitual seizures. In these five patients, part of the typical seizure onset symptoms were correlated with an initial ictal activity originating in the SMA, while the rest of the symptom- 
atology varied in accordance with the other cortical areas pertaining to the EZ. No seizures with very clear cut onset solely in the SMA were recorded, as the ictal propagation usually involved the middle cingulate cortex and/or the premotor cortex in less than 10 milliseconds, as well as other frontal and parietal cortical structures, depending on epilepsy duration and age at onset.

The spontaneous individual seizure manifestations in each of the five patients is presented in detail in Table 1.

Auras could be identified in four patients and were non-specific (falling sensation, dizziness, panic, restlessness, palpitations, headache, and warmth). Fear and sensory perceptions (shiver, alien limb sensation and paresthesias) were reported by three of the patients, out of which two had left SMA and middle cingulate cortex as EZs. In the case of M.M., the patient described rare occasions in which he felt that his right upper limb disappeared or that it did not belong to him (alien limb sensation) just before the onset of the seizure. Similar, patient V.A.D. described different auras, most prominent being paresthesia in either the upper or lower limb. In both patients, the EZ was correctly lateralized through semiology.
Consciousness during seizures was usually altered when the epileptogenic zone involved the dominant hemisphere. Although frequent seizures and with definite focal onset, all patients experienced generalization at some point in their personal history.

At the onset of the seizure, grimacing was common for most patients, but with poor correlation with the seizure onset hemisphere of origin. Head and eyes version occurred in 4 out of 5 patients, but to both sides, failing in lateralization of the EZ. The movements of the limbs were either tonic extension, dystonic posturing or upper limbs automatisms, with no lateralizing value. When observing the presence of any asynchronous movement, earlier or more prominent, most patients had upper limb dystonic posturing contralateral to the seizure generating hemisphere.

Vegetative manifestations during the seizures included face cyanosis, hypersalivation and dyspnea in 4 out of 5 patients, which would be expected when the epileptogenic network is widely distributed.

Seizure duration was similar, with three of the patients having the length of the seizures of up to 40 seconds. They have in common the inclusion

TABLE 1. Typical seizure semiology description in the selected patients, in which the EZ included the SMA

\begin{tabular}{|c|c|c|c|c|c|c|c|c|c|c|}
\hline Patient & Gender & Age & $\begin{array}{l}\text { Subjective } \\
\text { symptoms }\end{array}$ & Face & Head and eyes & Limbs & Consciousness & Vegetative & $\begin{array}{l}\text { Genera- } \\
\text { lization }\end{array}$ & EZ \\
\hline O.H. & $M$ & 29 & \begin{tabular}{|} 
- fear sensa on \\
- ascending \\
epigastric sensa on \\
- palpita ons
\end{tabular} & $\begin{array}{c}\text { - grimace with } \\
\text { mouth drooping } \\
\text { on the } \mathrm{R} \\
\text { - locked jaw }\end{array}$ & $\begin{array}{c}\text { - fixed gaze } \\
\text { - version to the } \\
\text { R or } L \text { side }\end{array}$ & $\begin{array}{l}\text { - dystonic } \\
\text { posturing of LUL, } \\
\text { then LLL or RUL }\end{array}$ & preserved & $\begin{array}{c}\text { hyper- } \\
\text { saliva on, } \\
\text { face cyanosis }\end{array}$ & yes & $\begin{array}{l}\text { Right SMA, } \\
\mathrm{MCC}, \mathrm{PCL}, \\
\text { OpR }\end{array}$ \\
\hline M.M. & $M$ & 28 & \begin{tabular}{|c|} 
- alien limb \\
sensa on of RUL or \\
all limbs \\
- autoscopy \\
- dizziness
\end{tabular} & None & $\begin{array}{c}- \text { version to the } \\
L \text { and up }\end{array}$ & $\begin{array}{c}\text { - dystonic } \\
\text { posturing of BUL } \\
\text { - tonic extension } \\
\text { of RUL } \\
\text { - BUL } \\
\text { automa sms }\end{array}$ & altered & none & yes & $\begin{array}{l}\text { Le SMA, } \\
\text { MCC }\end{array}$ \\
\hline P.D. & $\mathrm{F}$ & 36 & $\begin{array}{c}\text { - ascending cervical } \\
\text { shiver }\end{array}$ & $\begin{array}{l}\text { - grimace more } \\
\text { on the } L\end{array}$ & $\begin{array}{l}\text { - palpebral } \\
\text { clonias }\end{array}$ & $\begin{array}{c}-\mathrm{BUL} \\
\text { automa sms }\end{array}$ & $\begin{array}{c}\text { preserved, } \\
\text { rarely altered }\end{array}$ & dyspnea & yes, rare & $\begin{array}{l}\text { Le SMA, } \\
\text { MCC }\end{array}$ \\
\hline R.I.A. & $\mathrm{F}$ & 16 & None & $\begin{array}{l}\text { - grimace with } \\
\text { mouth drooping } \\
\text { on the } \mathrm{R}\end{array}$ & $\begin{array}{c}\text { - fixed gaze } \\
\text { - version to the } \\
R>L\end{array}$ & $\begin{array}{c}\text { - dystonic } \\
\text { posturing of RUL } \\
-4 \text { sign } \\
\text { - tonic extension } \\
\text { of BUL }\end{array}$ & altered & $\begin{array}{c}\text { hyper- } \\
\text { saliva on } \\
\text { face cyanosis }\end{array}$ & yes & $\begin{array}{l}\text { Le SMA, } \\
\text { MCC, FEF, } \\
\text { PMC }\end{array}$ \\
\hline V.A.D. & M & 18 & $\begin{array}{l}\text { - paresthesias of } \\
\text { LUL or LLL } \\
\text { - sensa on he } \\
\text { needs to move }\end{array}$ & $\begin{array}{l}\text { - grimace } \\
\text { unspecific } \\
\text { - locked jaw }\end{array}$ & $\begin{array}{c}\text { - version to the } \\
L>R\end{array}$ & $\begin{array}{c}\text { - dystonic } \\
\text { posturing of BUL } \\
\text { or LUL } \\
\text { - tonic extension } \\
\text { of BUL } \\
\text { - anterior trunk } \\
\text { flexion } \\
\text { - LUL clonia }\end{array}$ & $\begin{array}{c}\text { preserved, } \\
\text { rarely altered }\end{array}$ & $\begin{array}{c}\text { face cyanosis } \\
\text { dyspnea }\end{array}$ & no & $\begin{array}{l}\text { Right SMA, } \\
\mathrm{MCC}, \mathrm{PCL}, \mathrm{S}\end{array}$ \\
\hline
\end{tabular}

Legend: EZ - epileptogenic zone, R - right, L - le , LUL - le upper limb, LLL le lower limb, BUL - both upper limbs, RUL - right upper limb, SMA - supplementary motor area, MCC - middle cingulate cortex, PCL - paracentral lobule, OpR - Rolandic operculum, FEF - frontal eye fields, PMC - premotor cortex, S-sensi ve cortex. 
(along with the SMA) of the middle cingulate cortex in the epileptogenic zone, which is known to rapidly spread impulses in the frontal brain areas and to the contralateral hemisphere. This could explain the reduced durations and speedy secondary generalization of the seizures.

Seizure frequency varied, the worst control under antiepileptic drugs being for the patient who had frequent seizures only during sleep (P.D.). As an observation, her seizures began 1-2 months after an accident without head injury, and her mother had similar manifestations during childhood after an accident, but only for a short timespan. Not all events had secondary generalization, the patient was usually conscious during seizures and could respond to questions or follow commands. In rare cases, the seizures caused unresponsiveness, ictal amnesia and postictal confusion.

During the direct cortical stimulation of the SMA, we obtained various responses, as can be observed in Table 2. Most responses to $50 \mathrm{~Hz}$ stimulation with low levels of injected currents involved the contralateral upper limb (clonia, abduction, flexion, tonic contraction) and the trunk, with a contraction of the thoracic muscles on the contralateral side. We obtained less consistent responses of the contralateral lower limb, such as clonia, flexion of the proximal part, or paresthesia of the distal part, and of the face (mouth contraction on the contralateral side). No speech impairment was obtained.

Table 3 illustrates the cortical structures that compose the EZ based on spontaneous seizure observation, as well as the results of the cortical stimulation with electrical pulses of $50 \mathrm{~Hz}$ at various current intensities. The final resection area was decided using the electro-anatomo-clinical correlation of all available information. All patients had a good post-surgery outcome (Engel class I and II).

In 3 out of 5 patients, the scalp EEG interictal discharges were consistent with the EZ lateralization, while in the other two patients the discharges were most prominent over the midline. Most valuable for the good outcome of the resection were, as expected, the spontaneous SEEG seizures, showing the $\mathrm{EZ}$ at seizure onset and the structures to which the early spreading of the ictal activity occurred. The $50 \mathrm{~Hz}$ stimulation determined either typical seizures and/or seizure sensation in the EZ brain structures, but also in adjacent areas, which were only involved in the later spreading of the seizure.

The most important connections, observed as statistically significant SPES early responses to SMA stimulation in our five patients, were with the

TABLE 2. Clinical responses $50 \mathrm{~Hz}$ stimulation of the SMA

\begin{tabular}{|c|c|c|c|c|c|c|c|}
\hline Patient & Gender & EZ & Effect side & Upper limb & Trunk & Lower limb & Grimace \\
\hline O.H. & M & R & L & $\begin{array}{c}\text { clonia, abduc on, tonic } \\
\text { contrac on }\end{array}$ & flexion & - \\
\hline M.M. & M & L & R & abduc on, flexion & flexion & clonia, distal paresthesia & - \\
\hline P.D. & F & L & R & abduc on & flexion & clonia & - \\
\hline R.I.A. & F & L & R & $\begin{array}{c}\text { abduc on, flexion, tonic } \\
\text { contrac on }\end{array}$ & - & mouth \\
V.A.D. & M & R & L & abduc on, flexion & flexion & clonia, extension, exterior rota on & \\
\hline
\end{tabular}

TABLE 3. Electric characteristics of the epileptogenic zone during scalp videoEEG and SEEG recordings.

\begin{tabular}{|l|l|l|l|l|l|}
\hline Patient & $\begin{array}{l}\text { Spontaneous SEEG } \\
\text { seizures }\end{array}$ & $\begin{array}{l}\text { Scalp EEG interictal } \\
\text { discharges }\end{array}$ & $\begin{array}{l}\text { Provoked seizures } \\
\text { (50 Hz stimulation) }\end{array}$ & $\begin{array}{l}\text { Seizure sensation without } \\
\text { seizure (50 Hz stimulation) }\end{array}$ & Resection \\
\hline O.H. & $\begin{array}{l}\text { R SMA, R PMC, R OpR, } \\
\text { R MCC, R PCL }\end{array}$ & Fp2, F4, F8, C4, Fz & R SMA, R MCC, R PMC & R preSMA, R MCC, R PMC & $\begin{array}{l}\text { R SMA, R MCC, R OpR, } \\
\text { R PCL, R PMC, R SPL }\end{array}$ \\
\hline M.M. & L SMA, L MCC & $\begin{array}{l}\text { Fp1, F3, F4,F7, F8, C4, } \\
\text { Fz, Cz }\end{array}$ & L preSMA, L dIPFC & L MCC, L PCL, R dIPFC & $\begin{array}{l}\text { L preSMA, L SMA, } \\
\text { L MCC }\end{array}$ \\
\hline P.D. & L SMA, L MCC & $\begin{array}{l}\text { Fp1, Fp2, F3, F4,F8, } \\
\text { C4, T4, Fz, Cz }\end{array}$ & L SMA & L preSMA, L MCC, R PrC & L SMA, L MCC \\
\hline R.I.A. & $\begin{array}{l}\text { L SMA, L FEF, L PMC, } \\
\text { L MCC }\end{array}$ & Fp1, F3, C3, Fz & $\begin{array}{l}\text { L SMA, L PMC, } \\
\text { L dmPFC, R dmPFC }\end{array}$ & L dIPFC, R PMC & $\begin{array}{l}\text { L SMA, L FEF, L PMC, } \\
\text { L MCC }\end{array}$ \\
\hline V.A.D. & $\begin{array}{l}\text { R SMA, R MCC, R PCL, } \\
\text { R S }\end{array}$ & F4, C4, Fz, Cz & R MCC, R PCL, R inferior S & $\begin{array}{l}\text { R SMA, R MCC, R PCL, } \\
\text { R inferior S, R OpR }\end{array}$ \\
\hline
\end{tabular}

Legend: R - right ; L - le ; SMA - supplementary motor area; $M C C$ - middle cingulum; PMC - premotor cortex; FEF - frontal eye fields; dmPFC - dorsomedial prefrontal cortex; dIPFC - dorsolateral prefrontal cortex; $\mathrm{PCL}$ - paracentral lobule; $\operatorname{PrC}$ - precuneus; $\mathrm{SPL}$ - superior parietal lobule; OpR - Rolandic operculum; OpS - sensi ve operculum; 
ipsilateral middle cingulate cortex (MCC), premotor cortex (PMC), dorsolateral prefrontal cortex (dlPFC), primary motor area (R), primary sensitive area (S), frontal eye fields (FEF), paracentral lobule (PCL), and the contralateral SMA. There was only one constant connection in all patients, between the SMA and the MCC.

One patient (V.A.D.) had SPES stimulation of the SMA during both wakefulness and sleep. Fig. 1 depicts the statistically significant variation of connections of the SMA. Only few connections exhibited a marked decrease during sleep, between the SMA and superior parietal lobule (SPL), dIPFC and within itself. The connection with the PMC and posterior cingulate cortex (PCC) were markedly enhanced during sleep. The rest of the connections had little variation and indicate no significant modulatory effect of sleep.

All five patients had the resection of the identified epileptogenic zone and it included the SMA and MCC. In the first days after the intervention, all patients had a minor or moderate hemiparesis of the contralateral limbs and the patients with left hemisphere resection had motor aphasia. These neurological deficits resolved within 7 days. All patients experienced a marked seizure frequency and severity reduction, four patients with Engel I outcome and one with Engel II.

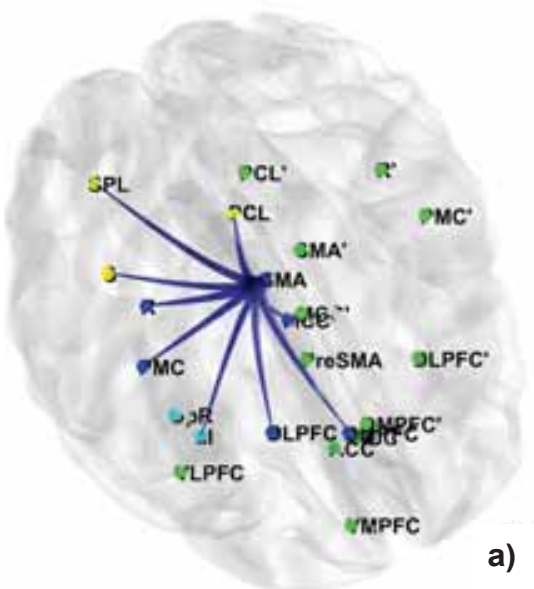

a)

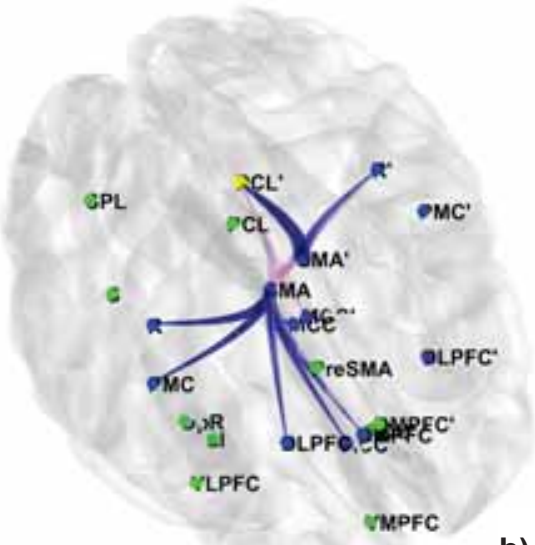

b)

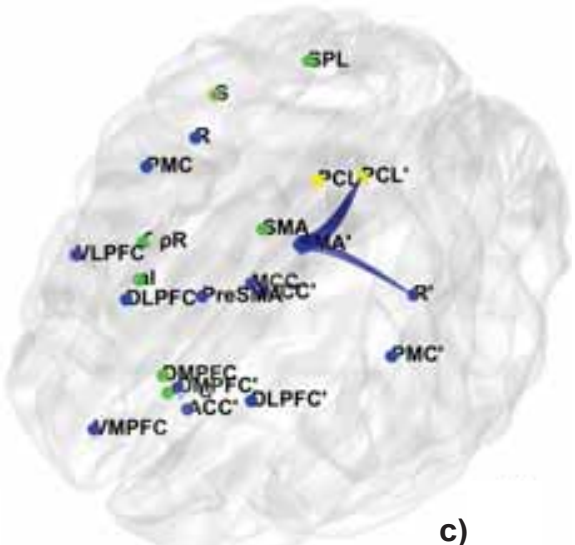

c)
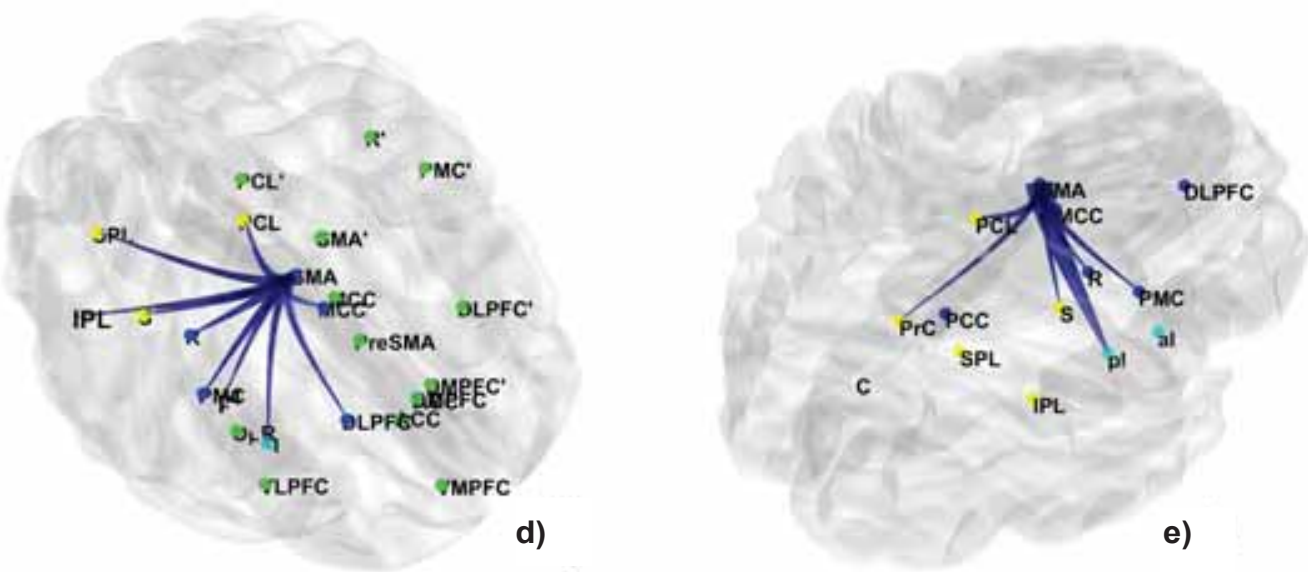

FIGURE 1. SMA statistically significant connections during wakefulness (4 patients) and during sleep

(1 patient) as studied through SPES stimulation.

a) patient O.H.; b) patient M.M.; c) patient R.I.A, d) patient V.A.D. during wakefulness e) patient V.A.D. during sleep. As it can be observed, SMA connections during wakefulness are constant with the primary motor cortex (R), middle cingulate cortex (MCC), premotor cortex (PMC), dorsolateral prefrontal cortex (DLPFC), paracentral lobule (PCL), posterior cingulate cortex (PCC), sensitive cortex (S), part of the parietal cortex (superior or inferior parietal lobule; $S P L / I P L)$ as well as insular cortex (al, pl). Comparing d) and e), we observe that in patient V.A.D. sleep determines, in comparison with wakefulness, a lower number of significant connections of the SMA, favoring local connections (R, S, MCC, PMC, PCL, PrC - precuneus). Figure b) depicts bilateral connectivity and shows an important relationship between right SMA and left SMA (SMA'), but not in a), c) and d), suggesting it is an effect of the particular epileptogenic network of patient M.M. 


\section{DISCUSSIONS}

Because the EZ structures included mostly deep situated brain areas, standard scalp EEG recordings could not accurately identify the restricted seizure generating areas, thus the necessity of intracerebral electrodes implantation. The SEEG recordings along with direct electrical stimulations permitted the delineation of surgically removable zones with minimum deficit.

Clinical characteristics of seizures, with specific motor movements suggesting SMA involvement (dystonic posturing of the upper limbs, grimace, head and eye version, trunk flexion), and other ictal manifestations, provided only cues to lobar localization of the EZ, but inconsistent lateralization, confirming previous studies.

SEEG recordings delineated an extended and complex epileptogenic network, with an important connection between SMA and MCC, the ictal activity onset being concomitant. In the studied lot, the epileptogenic zones also included other mesial frontal or parietal structures in the early seizure onset, probably due to the long timespan that permitted the evolution of the epileptogenic network. The spreading of seizures was fast, probably due to the early implication of the cingulate gyrus, also facilitating contralateral spreading.

Direct electrical stimulation identified only motor effects, but no speech arrest or elementary sensations as were described in other studies. This might be the consequence of different stimulation protocols and injected current charge. We chose not to repeat the stimulation with higher current charge if we obtained a response, although maybe this restricted the number of overall observed clinical effects. SPES stimulation successfully identified connectivity of the SMA without producing clinical symptoms and demonstrated a modulatory influence of sleep on certain connections, a novel finding in the current literature. Further studies on larger lots of patients are needed.

An important observation is that an epileptogenic zone that involves parietal and frontal mesial gyri, such as the paracental lobule, cingulum and SMA, can determine typical frontal seizure manifestations, such as the case of patient V.A.D. Successful surgical treatment also required parietal mesial structures resection. This promotes caution in the interpretation of seizure semiology and characteristics.

The surgical outcomes are consistent with previous studies regarding resection of the SMA and MCC in patients with tumors in these areas, but with slightly better long term results (Kim et al., 2013).

\section{CONCLUSIONS}

Supplementary motor area as part of the epileptogenic zone gives rise to a wide variety of manifestations, while awake or during sleep. Lateralization from the semiology of the seizures is not always reliable. In the patients we studied, auras also had little lateralizing value. The shorter the seizures, the more difficult to identify lateralizing signs.

Intracerebral depth electrodes implantation proved, in all cases, very useful for the accurate identification of epileptogenic zones, even in the most difficult cases, with nonspecific seizure semiology and false lateralization.

From SEEG recordings, we concluded that the SMA has widespread connections with the rest of the frontal lobe and parietal lobe. The most important relationships were with the premotor cortex and the middle cingulate cortex, which require inclusion into the final resection for a good outcome.

Direct electrical stimulation of the SMA usually generates motor effects on the contralateral limbs. We did not observe speech arrest or elementary sensations. Sleep seems to have a modulatory effect on SMA connectivity, but further studies are needed.

Resection of the SMA does seem not cause long term deficits and is safe to cut out when it is part of the epileptogenic zone in order to reduce or stop seizure occurrence.

\section{Acknowledgments:}

SEEG procedures and data collection were supported by Romanian UEFISCDI research grant PNII-ID-PCE-2011-3-0240.

We present or special thanks to our team members in the National Romanian Epilepsy Surgery Programme, without which this research and article would have not been possible: Andrei Barborica and Cristian Donos (University of Bucharest, Phys- 
ics Faculty), Jean Ciurea and Alin Rasina (Bagdasar Arseni Emergency Hospital, Neurosurgery Clinic), Andrei Daneasa, Mihai Maliia, Victorita Raiciu,
Mariana Popa, Mariana Popescu (Univseristy Emergency Hospital Bucharest).

Conflict of interest: none declared

\section{REFERENCES}

1. Bonelli S.B. \& Baumgartner C. (2002). [Frontal lobe epilepsy--clinical seizure seminology]. Wien Klin Wochenschr, 114(8-9), 334-340. Retrieved from http://www.ncbi.nlm.nih.gov/pubmed/12212369

2. Bonelli S.B., Lurger S., Zimprich F., Stogmann E., Assem-Hilger E., \& Baumgartner C. (2007). Clinical seizure lateralization in frontal lobe epilepsy. Epilepsia, 48(3), 517-523. http://doi. org/10.1111/j.1528-1167.2006.00943.x

3. Bonini F., McGonigal A., Trébuchon A., Gavaret M., Bartolomei F., Giusiano B., \& Chauvel P. (2014). Frontal lobe seizures: from clinical semiology to localization. Epilepsia, 55(2), 264-77. http://doi. org/10.1111/epi.12490

4. Chassagnon S., Minotti L., Kremer S., Hoffmann D., \& Kahane P. (2008). Somatosensory, motor, and reaching/grasping responses to direct electrical stimulation of the human cingulate motor areas. Journal of Neurosurgery, 109(4), 593-604. http://doi.org/10.3171/ JNS/2008/109/10/0593

5. Filevich E., Kühn S., \& Haggard P. (2012, November 1). Negative motor phenomena in cortical stimulation: Implications for inhibitory control of human action. Cortex. Elsevier. http://doi.org/10.1016/j. cortex.2012.04.014

6. Janszky J., Fogarasi A., Jokeit H., \& Ebner A. (2001). Lateralizing value of unilateral motor and somatosensory manifestations in frontal

lobe seizures. Epilepsy Res, 43(2), 125-133. Retrieved from http:// www.ncbi.nlm.nih.gov/pubmed/11164701

7. Kim Y.-H., Kim C.H., Kim J.S., Lee S.K., Han J.H., Kim C.-Y., \& Chung C.K. (2013). Risk factor analysis of the development of new neurological deficits following supplementary motor area resection. Journal of Neurosurgery, 119(1), 7-14. http://doi. org/10.3171/2013.3.JNS121492

8. Saint-Hilaire J.M. \& Lee M.A. (2000). Localizing and lateralizing value of epileptic symptoms in temporal lobe epilepsy. Can J Neurol Sci, 27 Suppl 1, S1-5-1. Retrieved from http://www.ncbi.nlm.nih.gov/ pubmed/10830319

9. Serles W., Pataraia E., Bacher J., Olbrich A., Aull S., Lehrner J., Baumgartner C. (1998). Clinical seizure lateralization in mesial temporal lobe epilepsy: differences between patients with unitemporal and bitemporal interictal spikes. Neurology, 50(3), 742-747. Retrieved from http://www.ncbi.nlm.nih.gov/pubmed/9521267

10. Tufenkjian K., \& Lüders H.O. (2012). Seizure semiology: its value and limitations in localizing the epileptogenic zone. Journal of Clinical Neurology (Seoul, Korea), 8(4), 243-50. http://doi.org/10.3988/ jcn.2012.8.4.243 